by administration of L. murinus. They then showed that one indole metabolite downregulated differentiation of $\mathrm{T}$ lymphocytes into $\mathrm{T}_{\mathrm{H}} 17$ cells.

Do these observations translate to humans? Wilck et al. gave 12 healthy people $6 \mathrm{~g}$ of supplemental sodium chloride per day for 14 days. The subjects' mean blood pressure increased over time, as did the numbers of $\mathrm{T}_{\mathrm{H}} 17$ cells in their blood. In four of the five individuals who had Lactobacillus species in their guts before treatment, five species became undetectable after two weeks. Ten of the people gained extra Lactobacillus species during treatment, but overall Lactobacillus abundance declined. The authors drew control data from previous studies.

Wilck and colleagues' animal analysis, like many such studies, has provided key insights and raised compelling questions about the role of the microbiota in regulating host traits. However, there are several caveats when considering whether these findings will translate to humans. First, the authors gave mice $0.3 \mathrm{~g}$ of salt daily, which is a 15-fold increase from their normal intake. This high-salt environment would be difficult to create in humans because the equivalent daily dose for a $70-\mathrm{kg}$ human, scaled by surface area, would be roughly $170 \mathrm{~g}$ - much more than humans could tolerate. Furthermore, most dietary sodium in humans is typically absorbed in the small intestine ${ }^{10}$. Thus, if elevated salt intake has direct effects on the gut microbiota, these effects probably occur before nutrients reach the colon, which was studied here.

Second, Wilck and colleagues' human data concern a specific scenario - people with normal blood pressure on an HSD for a relatively short time. This scenario and these findings are not necessarily informative about people who have chronic hypertension, particularly those with other underlying health problems, because of additional confounding factors.

Finally, the authors did not analyse whether changes in the abundance of Lactobacillus species correlated with the magnitude of changes in blood pressure, which could have helped to show a causal relationship between these factors. A larger study that includes people with normal and high blood pressure, compares various salt doses and a placebo, and takes functional readouts of the microbiota over time is eagerly awaited.

The list of complex diseases in which the gut microbiota might play a part is growing, but careful attention to interacting co-factors is needed when testing hypotheses. For instance, when considering a proposed role ${ }^{11}$ for the microbiota in cardiovascular disease, several variables must be taken into account. Gut microbes produce the molecule trimethylamine from dietary compounds found in red meat, and the liver converts it to trimethylamine oxide (TMAO). In animals, increases in TMAO promote a condition called atherosclerosis ${ }^{11}$, which increases the risk of heart attack and stroke. However, TMAO production is not necessary for the development of atherosclerosis, and other factors are required in addition to gut microbes for TMAO production. Thus, the gut microbiota contributes to atherosclerosis, but only in individuals who have certain combinations of microbes, diet and other risk factors.

Should hypertension be added to the list of conditions promoted by the gut microbiota? Future studies will no doubt tell. If this connection is real, one might predict - because of other variable factors - that the effects will be modest and restricted to a subset of individuals. Nonetheless, even modest effects are more than worthy of further study, because of the profound potential for global health benefits.

David A. Relman is in the Departments of Medicine, and of Microbiology \& Immunology, Stanford University, Stanford, California 94305-5124, USA, and at the Veterans Affairs Palo Alto Health Care System,
Palo Alto, California.

e-mail:relman@stanford.edu

1. Institute of Medicine. A Population-Based Policy and Systems Change Approach to Prevent and Control Hypertension (Natl Acad. Press, 2010).

2. Wilck, N. et al. Nature 551, 585-589 (2017).

3. US Department of Health and Human Services, US Department of Agriculture. What We Eat in America. NHANES 2011-2012. Available at http://www.ars. usda.gov/ARSUserFiles/80400530/pdf/1112/ tables_1-40_2011-2012.pdf

4. Institute of Medicine. Dietary Reference Intakes for Water, Potassium, Sodium Chloride, and Sulfate (Natl Acad. Press, 2005).

5. Kotchen, T. A., Cowley A. W. Jr \& Frohlich, E. D. N. Engl. J. Med. 368, 1229-1237 (2013)

6. Kleinewietfeld, M. et al. Nature 496, 518-522 (2013).

7. Madhur, M. S. et al. Hypertension 55, 500-507 (2010).

8. Burkett, P. R., Meyer zu Horste, G. \& Kuchroo, V. K J. Clin. Invest. 125, 2211-2219 (2015).

9. Rothhammer, V. et al. Nature Med. 22, 586-597 (2016).

10.Sandle, G. I. Gut 43, 294-299 (1998).

11.Tang, W. H. W. \& Hazen, S. L. J. Clin. Invest. 124 4204-4211 (2014).

This article was published online on 15 November 2017.

\title{
ELECTRONICS
}

\section{Contactless health-care sensing}

A system for monitoring a person's vital signs - such as their blood pressure and heart rate - offers many advantages over currently used technologies, including enhanced sensing capabilities, comfort and convenience.

\section{LEENA UKKONEN \& LAURI SYDÄNHEIMO}

S ystems that can track a person's vital signs are of paramount importance for medicine and health care. Vital signs include heart rate, blood pressure, respiration rate and breathing rate. Tracking systems that are both wearable and wireless are desirable because they are minimally intrusive $e^{1,2}$ and could allow institutionalized care to be replaced with care at home for the elderly ${ }^{3}$. Writing in Nature Electronics, Hui and $\mathrm{Kan}^{4}$ report such a system, which could find applications in both home care and clinical facilities.

A variety of technologies are currently used to monitor vital signs. They include electrodes, stethoscopes, strain gauges and ultrasound devices, each of which has drawbacks in terms of comfort and convenience. For instance, electrodes (such as electrocardiograms) and stethoscopes require direct skin contact, which can cause discomfort and restrict a person's movement. Strain gauges monitor blood pressure and respiration using belts or cuffs, which can disrupt everyday activities. Finally, ultrasound devices are portable, but are often rather cumbersome.

An alternative approach is to use radio-frequency backscattering, in which radio waves are reflected off the body and then detected ${ }^{5}$. A clear advantage of this method is that it does not require direct skin contact. The radiofrequency technologies that are currently used can monitor breathing, but struggle to detect small mechanical vibrations inside the body, such as a person's heartbeat or pulse ${ }^{6}$. Although heart rate can be extracted using careful filtering methods, measuring blood pressure and monitoring many people simultaneously has not been possible.

Hui and Kan's health-care system combines radio-frequency backscattering with a technique that they call near-field coherent sensing (NCS). In NCS, radio waves are directed into the body's tissues. This produces stronger backscattered signals from internal organs than signals obtained using conventional methods, in which the waves are reflected mainly off the body's surface ${ }^{6,7}$. Furthermore, because the wavelength of a propagating wave is shorter inside the body than outside it, NCS is more sensitive to the body's mechanical 


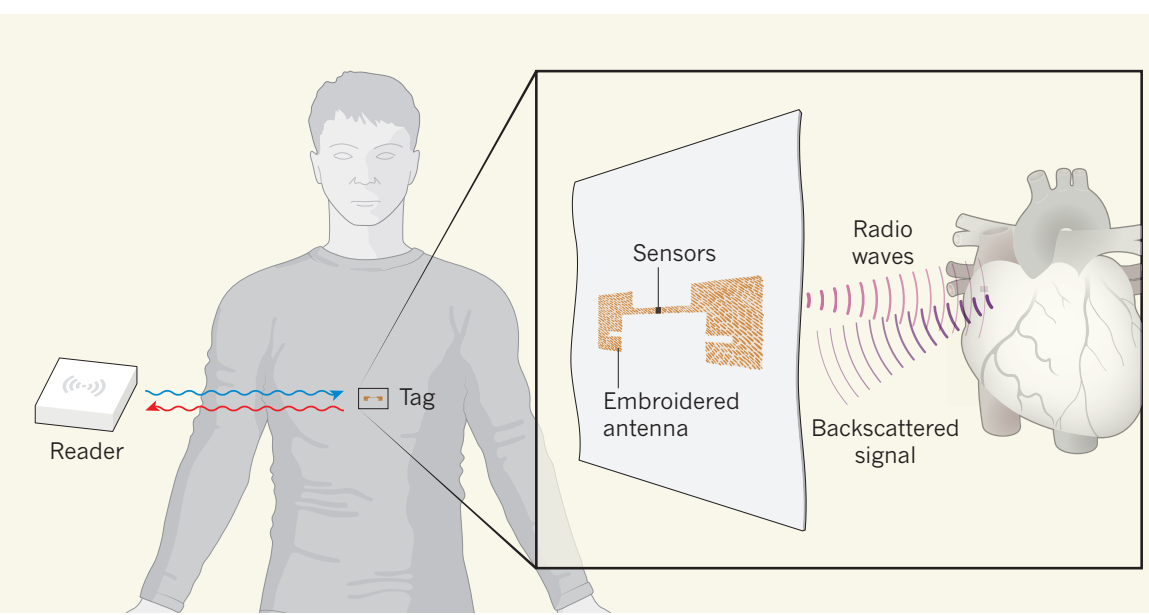

Figure 1 | A wireless health-care system. Hui and $\mathrm{Kan}^{4}$ report a system for monitoring a person's vital signs, such as their heart rate. The system is wireless and does not require skin contact. It uses a device known as a passive radio-frequency identification tag, which can be integrated into clothing. The tag comprises an embroidered antenna and sensors, and is powered by electromagnetic energy (blue arrow) from an electronic device called a reader. The antenna transmits radio waves that enter the body and are reflected. The backscattered signal is then picked up by the on-tag sensors. Finally, this information is sent to the reader (red arrow) to allow data on the person's vital signs to be retrieved.

motion than previous approaches.

To distinguish between backscattered signals associated with the various vital signs, it helps to know whether a particular signal originates from inside or outside the body. In the NCS method, the signals corresponding to internal motion are therefore combined (multiplexed), as are those associated with motion on the body's surface. Multiplexing is useful because it allows a group of independent signals to share a communication channel without interference.

Hui and Kan implemented their NCS technique using a device known as a passive radio-frequency identification tag, which is equipped with a unique digital identification $\operatorname{code}^{8}$ (Fig. 1). The authors' tag comprises an antenna and sensors, and is powered by the electromagnetic energy from an external device called a reader. When the tag is activated, the antenna transmits radio waves on and into the body, and the backscattered signals are picked up by the sensors. This information is then sent to the reader to allow data on the body's vital signs to be retrieved. The authors show that their tag can be embroidered directly into clothing. Even when the material was laundered several times, the integrated tag retained its geometry and functionality.

Another advantage of the NCS method is that the downlink (the communication link from the reader to the tag) and the uplink (the link from the tag to the reader) can be separated from each other. The authors' health-care system can therefore detect signals more effectively than currently used methods ${ }^{6,7}$. The tag is a passive backscatterer (it communicates by reflecting wireless signals), which means that it can use existing wireless communication technology. The digital information that is transferred between the reader and the tag can include the tag identification code and additional information from the on-tag sensors.

Hui and Kan point out that, to maximize the distance between the tag and the reader across which measurements can be made, and to reduce the impact of the person's movement, an active radio-frequency identification tag could instead be used. These tags are battery-powered and continuously emit radio waves, which means that they do not need to receive electromagnetic energy from a reader. However, active tags are generally much more expensive than passive tags.

Hui and Kan's system can monitor many

\section{MICHELLE ELLIOTT}

$\mathrm{U}$ nderstanding the processes that broaden the gap between the wealthy and the poor is a concern for social scientists and policymakers worldwide ${ }^{1}$. Although economists and historians can identify key factors that drive inequality today, it is less clear where its ultimate origins lie and what dynamics first led to economic people simultaneously, and could lead to the automation of vital-sign monitoring in care facilities. However, the system will need a reader device that is compact, portable and easily integrated into such environments. This is one of the main challenges that must be overcome before the system can be used in a real-world setting. Future work will also need to test the reliability of such wearable, textilebased devices, and to demonstrate that they are effective in a clinical study.

Wireless health technologies will become increasingly important in the future, particularly given the world's ageing population. Hui and Kan's work provides a platform on which to create contactless, wearable devices for various health-care and health-promotion applications.

Leena Ukkonen and Lauri Sydänheimo are in the Faculty of Biomedical Sciences and Engineering, Tampere University of Technology, 33720 Tampere, Finland. e-mails:leena.ukkonen@tut.fi; lauri.sydanheimo@tut.fi

1. Pantelopoulos, A. \& Bourbakis, N. G. IEEE Trans. Systems Man Cybernet. C 40, 1-12 (2010).

2. Mahdavi, H. \& Rosell-Ferrer, J. Physiol. Meas. $\mathbf{3 8}$ 1226-1241 (2017).

3. Teixeira, C. C. et al. http://dx.doi.org/10.1590/0104 0707201500003970014 (2015)

4. Hui, X. \& Kan, E. C. Nature Electron. http://dx.doi. org/10.1038/s41928-017-0001-0 (2017).

5. Lin, J. C. Proc. IEEE 63, 1530 (1975).

6. Kao, T.-Y. J. et al. IEEE Trans. Microw. Theory Techniques 61, 1649-1659 (2013)

7. Chen, K. M., Huang, Y., Zhang, J. \& Norman, A. IEEE Trans. Biomed. Eng. 47, 105-114 (2000).

8. Catarinucci, L., Colella, R. \& Tarricone, L. 3rd Int. Symp. Appl. Sci. Biomed. Commun. Technol. http://dx.doi.org/10.1109/ISABEL.2010.5702838 (2010).

This article was published online on 27 November 2017

\section{Inequality has deep roots in Eurasia}

\section{A study of 64 archaeological sites across four continents shows that the growth of agricultural and political systems provoked economic disparities, more so in Eurasia than in North America. SEE LETTER P.619}

differences ${ }^{2}$. Archaeology is in a unique position to address this issue, because it can be used to study multiple ancient civilizations over long periods of time and before the existence of written records. On page 619, Kohler et al. ${ }^{3}$ present an archaeological study that provides evidence that increasing dependence on agriculture and the growing complexity of political systems intensified wealth inequalities over time. 